\title{
ANTARES and KM3NeT programs for the supernova neutrino detection
}

\author{
Vladimir Kulikovskiy \\ on behalf of the ANTARES and KM3NeT collaborations
}

Aix Marseille Univ, CNRS/IN2P3, CPPM, Marseille, France

email: kulikovskiy@cppm.in2p3.fr

\begin{abstract}
The currently working ANTARES neutrino telescope has capabilities to detect neutrinos produced in astrophysical transient sources. Neutrino alerts are regularly generated to trigger multi-wavelength observatories. Potential sources include gamma-ray bursts, core-collapse supernovae, and flaring active galactic nuclei. In particular, the neutrino detection together with the multi-wavelength observations may reveal hidden jets in the supernova explosions.

Supernovae remnants are currently the most promising acceleration sites of the cosmic rays in our Galaxy. The neutrino emission is expected during the cosmic ray interaction with the surrounding matter. The neutrino telescopes in the Northern hemisphere have excellent visibility to the most of the galactic supernovae remnants. Recent results on the search for point-sources with the ANTARES detector and the prospects for the future KM3NeT detector are presented.

Although ANTARES and KM3NeT detectors are mainly designed for high energy neutrino detection, the $\mathrm{MeV}$ neutrino signal from the supernova can be identified as a simultaneous increase of the counting rate of the optical modules in the detector. The noise from the optical background due to ${ }^{40} \mathrm{~K}$ decay in the sea water and the bioluminescence can be significantly reduced by using nanosecond coincidences between the nearby placed photomultipliers. This technique has been tested with the ANTARES storeys, each one consisting of three 10-inch photomultipliers, and it is further optimized for the KM3NeT telescope where the directional optical modules containing 313 -inch photomultipliers provide very promising expectations.
\end{abstract}

Keywords. neutrinos, supernovae: general, supernova remnants, instrumentation: detectors

\section{Introduction}

ANTARES and KM3NeT are neutrino telescopes presenting mainly three-dimensional photomultiplier (PMT) arrays in the deep sea water well shielded from atmospheric muons (more technical details will be given in Sec.2). Such telescopes can be used to study different evolution stages of the SuperNova (SN) explosions:

- Prompt SN emission (20 MeV neutrinos) should produce an increase of PMT rates in the detector. No event reconstruction but high sensitivity in time domain is expected for such observations. Simulations and search optimization for KM3NeT are presented in Sec. 3.

- Transient high energy neutrino emission ( $\mathrm{GeV}-\mathrm{TeV}$ e.g. neutrinos from GRB-SN, hidden jets) can be promptly detected by the neutrino telescopes (fast event reconstruction, neutrino direction and energy estimation) and the alerts can be forwarded to the followers. The existing and future programs are described in Sec. 4.

- Supernova Remnants can be efficient high energy hadron accelerators $(\sim \mathrm{GeV}-\mathrm{TeV}$ neutrinos). Searches of unknown steady point sources on the sky are performed with the long-term ANTARES data and planned with KM3NeT. They are enriched with searches 
through candidate catalogs (from gamma-ray observations mainly). Some ANTARES results and KM3NeT perspectives are summarized in Sec. 5.

\section{Technology and performance}

The ANTARES detector is the first undersea neutrino telescope. It is installed at $2475 \mathrm{~m}$ depth; its effective mass is about $10 \mathrm{Mt}$ and it is optimized for $E_{\nu} \gtrsim 10 \mathrm{TeV}$ (Ageron M. et al.(2011)). KM3NeT is a distributed research infrastructure that will host a high energy neutrino telescope (ARCA), offshore of Capo Passero, and an atmospheric neutrino detector (ORCA), offshore of Toulon in France (close to the ANTARES site), for the determination of the neutrino mass hierarchy (Adrián-Martínez S. et al. (2016)). ARCA has two detector blocks instrumenting about $1 \mathrm{Gt}$ and it is optimized for $E_{\nu} \gtrsim$ $10 \mathrm{TeV}$. Each detector block contains 115 detection units. Detection unit present a string with 18 optical modules. The ORCA detector has one detector block that instruments about 5.7 Mt optimized for $5-500 \mathrm{GeV}$ neutrinos. The total number of strings and optical modules in ORCA is the same as in one ARCA block, but the detector elements are placed closer to each other to improve the detector sensitivity at lower energies.

The KM3NeT detectors use new directional optical modules (DOMs) that host 31 3-inch PMTs. Each DOM has a total PMT photocathode effective area similar to the ANTARES storey with three 10-inch PMTs. Multi-PMT technology allows better light direction estimation and better optical background suppression by using time coincidence between PMT signals. PMTs and their digitization electronics as well as positioning/calibration instrumentation for each DOM are stored inside a 17-inch glass sphere (the same one that is used to store one ANTARES PMT). This concept allows furling of the detection unit for the deployment, which means more units deployment during each marine operation. Both detector sites have the underwater infrastructure ready for the detection units connection. Data taking at the ARCA site is ongoing with two detection units already.

Although being designed for the high energy neutrinos, both KM3NeT detectors are sensitive to the prompt $\mathrm{MeV}$ neutrinos from supernova explosions by observing the global light rate increase in the detector. More details are provided in the next section.

\section{Prompt emission detection}

Supernova fluxes simulation. The simulation of the supernova explosions are well advanced nowadays and reproduce the detected spectra and time distributions of the only so far existing SN1987A detection (for review see, for example, Mirizzi A. et al. (2016)). Variety of the models and parameters is present and used by different experiments for sensitivity estimations. Often, there is no overlap between the models used by different experiments, which complicates the direct comparison. In order to overcome this complexity a pragmatic approach is proposed in this work. In fact, it is very similar to the approach used by the JUNO collaboration (An F. et al. (2016)).

The SN neutrino spectrum is parametrized with the following function:

$$
f=E_{\nu}^{\alpha} e^{-(\alpha+1) E_{\nu} / \tilde{E_{\nu}}},
$$

which is defined with average energy $\tilde{E}_{\nu}$ and spectral shape/pinching parameter $\alpha$. As it was shown by Tamborra I. et al. (2012), the neutrino spectra are always fitted with parameters in the range of $2 \lesssim \alpha \lesssim 5$ or $0 \lesssim \eta \lesssim 4$.

Results of different supernova simulations predict about $15 \%-25 \%$ of the $\bar{\nu}_{e}$ emission in the first $100 \mathrm{~ms}$. The total $\nu$ flux considered by JUNO and generally accepted as a 
benchmark is $3 \times 10^{53}$ erg divided equally by flavours and $\nu / \bar{\nu}$. The more precise fraction of the $\bar{\nu}_{e}$ flux in total flux corresponds to $1 / 5 \cos ^{2} \theta_{12} \approx 0.14$, which includes oscillations in supernova mantle (Strumia A. and Vissani F. (2003)) and it is similar to $1 / 6$. The shape parameter $\alpha$ is around 3 during this interval and as also chosen by JUNO. The average energy for $\bar{\nu}_{e}$ is about $15 \mathrm{MeV}$. In JUNO three values are considered: $12 \mathrm{MeV}$, $14 \mathrm{MeV}$ and $16 \mathrm{MeV}$.

The cross-sections for inverse beta decay are taken from Strumia A. and Vissani F. (2003). The positron energy is calculated as:

$$
E_{e^{+}}^{C M}=\frac{m_{p}^{2}-m_{n}^{2}+m_{e}+2 E_{\nu} m_{p}}{2 \sqrt{m_{p}^{2}+2 m_{p} E_{\nu}}} \approx E_{\nu}-\frac{m_{n}^{2}-m_{p}^{2}-m_{e}^{2}}{2 m_{p}} \approx E_{\nu}-1.239 \mathrm{MeV},
$$

where $E_{\nu} \ll m_{p}$ is considered. As the Lorentz factor of the central mass frame is small for $E_{\nu} \ll m_{p}$ as well, $E_{e^{+}}^{C M} \approx E_{e^{+}}$(in the lab frame).

The positron distribution as a differential number of particles in 1 kton detector volume from as $\mathrm{SN}$ at $D=10 \mathrm{kpc}$ was calculated according to the following formula:

$$
d N_{e^{+}} / d E=\frac{1}{F} E_{\nu}^{\alpha} e^{-(\alpha+1) E_{\nu} / \tilde{E}_{\nu}}\left\{f_{100} \mathrm{~ms} \frac{E_{\bar{\nu}_{e}}^{\mathrm{tot}}}{\tilde{E}_{\nu}}\right\} \frac{1}{4 \pi D^{2}} \sigma_{I B D}\left\{M_{\mathrm{det}} / \mu_{\mathrm{H}_{2} 0} N_{\mathrm{A}} \times 2\right\}
$$

Where $1 / F$ is the normalization factor for the neutrino energy distribution, $f_{100 \mathrm{~ms}}=$ $25 \%$ used, $E_{\bar{\nu}_{e}}^{\text {tot }}=3 \times 10^{53} / 6$ erg. The first brackets present the calculation of the number of neutrinos at the source.

The previous analysis done for ANTARES (Kulikovskiy V. (2011)) used model 57 from Burrows A. (1988). According to this model, there are 65.7 positrons per kiloton of the detector in the first $100 \mathrm{~ms}$ and their spectrum follows the distribution described by the following parameterization:

$$
d N / d E \sim \frac{E[\mathrm{MeV}]^{2}}{1+e^{\frac{E[\mathrm{MeV}]-16}{6}}} .
$$

The distribution has shape similar to the previously described model with $\tilde{E}_{\nu}=16 \mathrm{MeV}$. Also the total numbers of positrons in 1 kton are similar: 45.7, 53.6 and 61.1 for $12 \mathrm{MeV}$, $14 \mathrm{MeV}$ and $16 \mathrm{MeV}$ correspondingly.

The positrons generator with the previously described parameterization was added to the GEANT 4 simulation of the optical modules (Hugon C. M. F. (2016)).

Background suppression with coincidences. The main optical background in the deep sea water arises from the ${ }^{40} \mathrm{~K}$ decay that goes mostly with $1.3 \mathrm{MeV}$ electron production. This background is well reproduced in GEANT 4 simulations and used for time and efficiency calibration of the detectors. Atmospheric muons represent also background for this measurement. Their contribution was taken from the data directly (Adrián-Martínez S. et al. (2014) and Adrián-Martínez S. et al. (2016b)). For simplicity, the muon rate for the ARCA site was taken as 1/4 of the muon flux at the ORCA site.

To suppress the background the coincidence method was developed for ANTARES (Kulikovskiy V. (2011)). Instead of all PMT signals, only coincident signals between two or three nearby PMTs (from the same storey) in a short time window ( $\sim 25 \mathrm{~ns}$ ) are used. The sensitivity estimations using these double and triple coincidence selections yield similar results (the sensitivity is about $5 \sigma$ for $\mathrm{SN}$ at $5 \mathrm{kpc}$ ). Since there are 31 nearby PMTs located in each DOM, higher coincidence level selections can be applied. The rate of hit selections with different coincidence levels in a single DOM is shown in Fig. 1 for ${ }^{40} \mathrm{~K}$ and muon backgrounds. For SN the constant emission during $100 \mathrm{~ms}$ is considered on the same plot for comparison. 


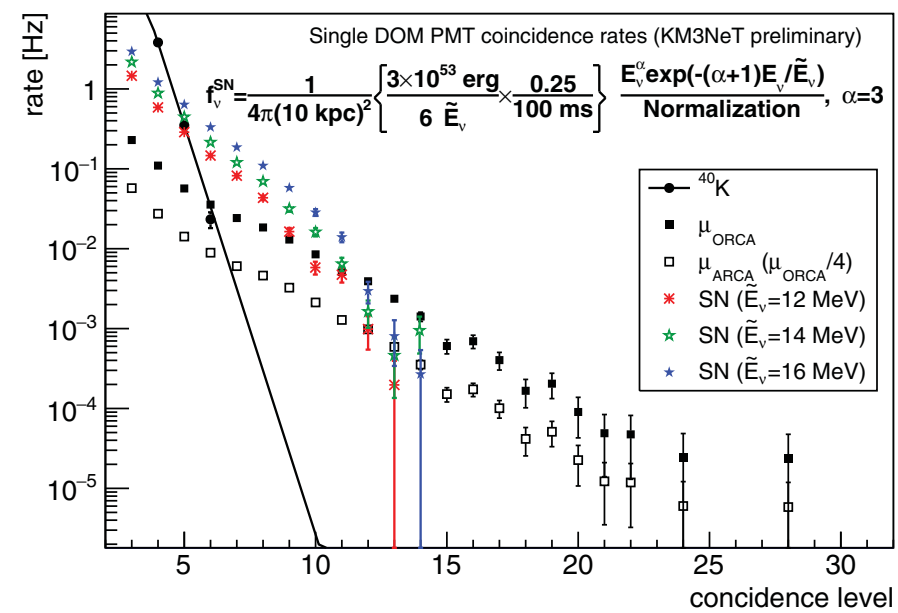

Figure 1. Coincidence event rates for the first $100 \mathrm{~ms}$ of the SN explosion (assuming constant emission during this period) for different mean neutrino energies. Main backgrounds $\left({ }^{40} \mathrm{~K}\right.$ decays and atmospheric muons) are also shown.

Table 1. Summary of the results on the SN prompt emission detection with KM3NeT.

\begin{tabular}{|c|c|c|c|c|c|c|c|}
\hline$\tilde{E}_{\bar{\nu}_{e}}$ & $\mathrm{MeV}$ & $\mid N_{\text {events }}$ & per block & $\mid D_{5 \sigma / 3 \sigma}$ & $(\mathrm{kpc})$ & $\mathbf{A R C A} \mid D_{5 \sigma / 3 \sigma}$ & $(\mathrm{kpc})$ ARCA \\
\hline | & 12 & | & 60 & | & $23 / 30$ & 1 & $16 / 20$ \\
\hline 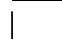 & 14 & 1 & 100 & 1 & $29 / 37$ & 1 & $19 / 25$ \\
\hline | & 16 & 1 & 150 & 1 & $37 / 47$ & 1 & $24 / 31$ \\
\hline
\end{tabular}

As it can be seen, the ${ }^{40} \mathrm{~K}$ decays produce in general events with fewer coincident hits in a DOM comparing to SN events. Muons produce even harder coincidence spectrum. This correlates with the lepton energies $\left(1.3 \mathrm{MeV} e^{-}\right.$for ${ }^{40} \mathrm{~K}, \gtrsim 10 \mathrm{MeV} e^{+}$for SN and $\mathrm{MeV}-\mathrm{GeV}$ energies for $\mu$ ) and suggests that coincidence level can be a rough event energy estimator.

Sensitivity estimation. For known signal and noise rates during a limited period sensitivity can be estimated as following:

$$
S=\frac{N_{\mathrm{DOMs}} \times T \times f_{\mathrm{SN}}}{\sqrt{N_{\mathrm{DOMs}} \times T \times f_{\mathrm{bg}}}}=\frac{f_{\mathrm{SN}}}{f_{\mathrm{bg}}} \sqrt{T \times N_{\mathrm{DOMs}}}
$$

The sensitivity decreases with source-detector distance as $1 / D^{2}$ following the positron production decrease with a distance (Eq. 3.3). For $\tilde{E}_{\nu}=16 \mathrm{MeV}$ model one can estimate the 5 sigma sensitivity till $36 \mathrm{kpc}$ for ARCA (all Milky Way) and $24 \mathrm{kpc}$ for ORCA (95\% of the Galactic progenitors). The complete set of results is summarized in Table 1.

These results are rather preliminary and conservative. In the next future time window optimization is planned; muon veto using coincidences between DOMs and topological features should increase sensitivity by increasing the signal/noise ratio.

\section{Transient high energy sources}

For transient and periodic sources the correlations in space and time between X-ray, optic and high energy gamma-ray telescopes and gravitational waves detectors restricts the background and boosts the sensitivities. A multi-wavelength follow-up program, denoted as TAToO (Telescopes-ANTARES Target of Opportunity), has operated within the 
ANTARES Collaboration since 2009. It is based on optical and, since mid 2013, X-ray follow-ups of selected high-energy neutrino events very shortly after their detection. The network is composed of the small robotic optical telescopes TAROT and ROTSE, and the Swift X-ray telescope (Adrián-Martínez S. et al. (2016b)). To be sensitive to all types of time variability in the astrophysical sources, the observational strategy is composed of a real-time observation for rapidly fading sources, such as Gamma Ray Burst afterglows, complemented by several observations during the following month specially adapted to detect the rising light curve of core collapse SNe. Unprecedented in this domain, the ANTARES telescope is able to generate alerts within a few seconds after the neutrino detection, and provides a precision on the reconstructed direction to better than $0.5^{\circ}$ at high energies $(E>1 \mathrm{TeV})$.

The exchange of alerts from the gamma-ray detectors is established through GCN $\dagger$. The ANTARES detector receives the GCN alert, which contains the position of the burst and its main features. The typical delay between the detection of a Gamma Ray Burst by the satellite and the time of the alert message distributed is around $10 \mathrm{~s}$. Buffered unfiltered data of about 2 minutes before the alert can be saved and analyzed afterwards using more efficient event reconstructions with a known direction (Albert A. et al. (2016)). That improves ANTARES sensitivity up to two times for $100 \mathrm{GeV}-1 \mathrm{TeV}$ neutrinos.

The future KM3NeT programs will follow the ANTARES developments extending them thanks to particular attention to the topic by the European Unionf.

\section{Steady sources detection}

Super novae remnants (SNRs) are one of the most promising candidates for the cosmic rays acceleration sites in our Galaxy. Debates about hadronic or leptonic acceleration mechanisms are still ongoing for many SNRs. Neutrino detection or strict upper limits can be an ultimate argument in such debates.

The structures of various SNRs defined with gamma ray telescopes cannot be resolved with current neutrino telescopes; they should appear as point-like or slightly extended spots in some cases. For the detection of neutrinos from the point-like sources, the most suited are the track-like events thanks to their sub-degree angular resolution. Atmospheric muon background should be suppressed. The event selection optimization was performed for the general point sources with $E_{\nu}^{-2}$ spectrum. For 3 years of ARCA $5 \sigma$ discovery potential fluxes are $E^{2} \Phi \approx 2 \cdot 10^{-9} \mathrm{GeV} \mathrm{cm}^{-2} \mathrm{~s}^{-1}$ for $\sin ($ declination) $<0.6$ (Fig. 2). This is more than one order of magnitude below the same discovery potential fluxes for IceCube with similar exposure at $\sin ($ declination $)<-0.2$. This difference grows up for the softer neutrino spectrums since the IceCube selection prefers higher event energies to suppress the atmospheric muon background in the Southern hemisphere. At high declinations track analysis loses its sensitivity due to atmospheric muon veto. Cascade events can recover sensitivity in this area. Event selection optimization including vertex containment inside the detector volume reaches $5 \sigma$ discovery potential at the level of $E^{2} \Phi \leqslant 5 \cdot 10^{-9} \mathrm{GeV} \mathrm{cm}^{-2} \mathrm{~s}^{-1}$ which is rather independent on the declination (Adrián-Martínez S. et al. (2016a)).

A separate analysis was done for the young shell-type SNR RXJ1713.7-3946. The neutrino spectrum used for the sensitivity estimation is derived from the measured

$\dagger$ gcn.gsfc.nasa.gov

$\ddagger$ ASTERICS-CLEOPATRA www.asterics2020.eu/cleopatra 


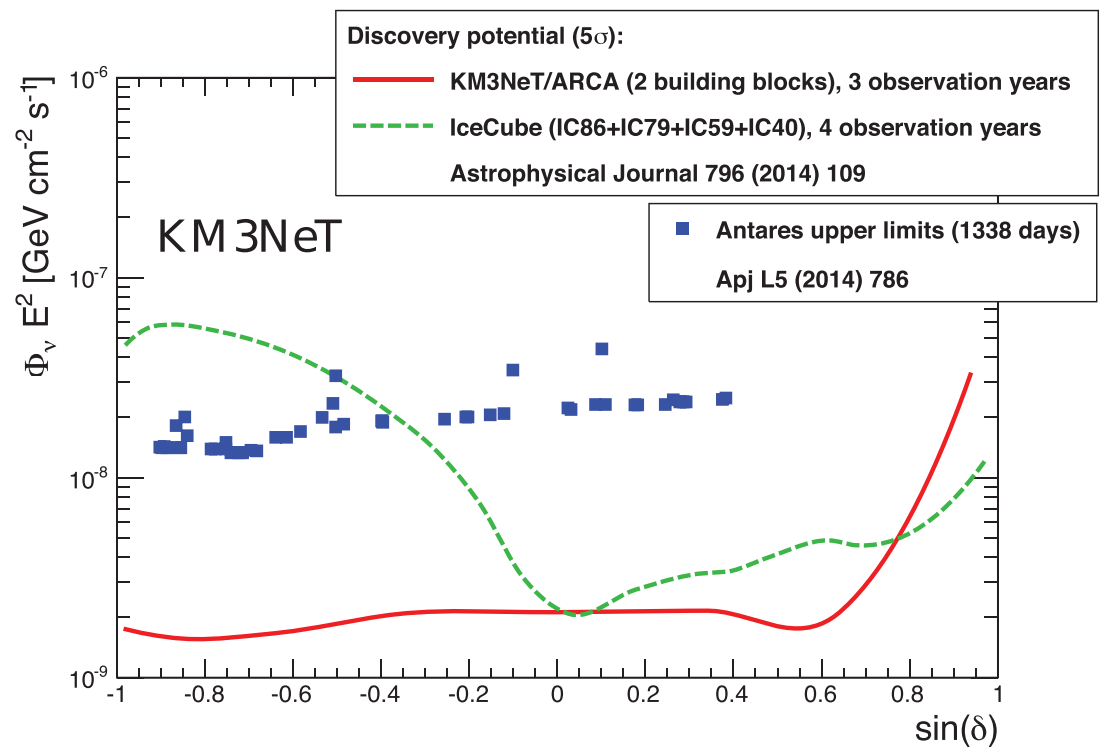

Figure 2. $5 \sigma$ discovery potential as a function of the source declination (red line) for one neutrino flavour, for point-like sources with a spectrum $\propto E^{-2}$ (ARCA, IceCube) and upper limits on particular sources for the ANTARES detector.

gamma-ray spectrum, which extends up to $100 \mathrm{TeV}$. The analysis adapted for the tracklike events reaches about $3 \sigma$ sensitivity in 4 years (Adrián-Martínez S. et al. (2016a)).

Recently, a new track reconstruction algorithm was developed. It provides more well reconstructed events and better neutrino angular resolution: $<0.1^{\circ}$ for $E>100 \mathrm{TeV}$ and almost reaches the intrinsic angle limit for $E<10 \mathrm{TeV}$. Also, different multivariate analysis tools are tested to discriminate the signal from two background components (atmospheric neutrinos and muons) which have different energy and angular spectra. In particular, Random Forests algorithms of Boosted Decision Trees perform well for this task. The first very preliminary results show that observation time may be reduced by one year to reach $3 \sigma$ discovery for RXJ1713 (Coniglione R. (2017)).

\section{References}

Adrián-Martínez, S. et al. 2016, J. of Phys. G 43084001

Ageron, M. et al. 2011, Nucl. Instrum. Meth. A 65611

Mirizzi, A. et al. 2016, Riv. Nuovo Cimento 391

An, F. et al. 2016, Phys. G 43030401

Tamborra, I. et al. 2012, Phys. Rev. D 86125031

Strumia, A. \& Vissani F. 2003, Phys. Lett. B 56442

Burrows, A. 1988, APJ 334891

Kulikovskiy, V. 2011, Proceedings of ICRC 2011 dx.doi.org/10.7529/ICRC2011/V07/1083

Hugon, C. M. F. 2016, PoS ICRC2015 1106

Adrián-Martínez, S. et al. 2014, Eur. Phys. J. C 743056

Adrián-Martínez, S. et al. 2016, Eur. Phys. J. C 7654

Adrián-Martínez, S. et al. 2016, JCAP 02062

Albert, A. et al. 2016, Accepted for publication to MNRAS, arXiv:1612.08589

Coniglione, R. 2017, Proceedings of XXV European Cosmic Ray Symposium arXiv:1701.05849 